with CHIKV IgM positive. In the EQ-5D (Figure 1), between $72 \%$ and $92 \%$ of patients didn't report problems on the mobility, self-care, usual activities, anxiety and depression dimensions. In contrast pain and discomfort dimensions were affected from moderate to extreme $(44.7 \%$ and 8.6\%). There weren't significant differences between true positives, true false, false negative within all the variables, except on the anxiety and depression dimensions where the false positives had a higher significant score in comparison to the other groups $(n: 10,38.5 \% ; p=0.00 ;$ OR:4.17, IC:1.8-9.57). In the EQ-5D-VAS, media for VAS was 75.56 (SD \pm 21.18 ) where the highest scores of VAS were found on the false negatives. In pain VAS, false positives as well as the duration greater than 7 weeks where associated with several pain (n: 10, 2.9\%; OR: 0.2; IC 1.25-6.33 y $n: 27,21.3 \% ; p=0.018 ;$ OR 2.02, IC: $1.12-3.66$ respectively)

Conclusion: In general, the disability of CHIKV infected patients was mild to moderate, finding the most affected dimensions to be pain, discomfort, anxiety and depression. The false positives and the population with anxiety and depression are related with higher score of disability.
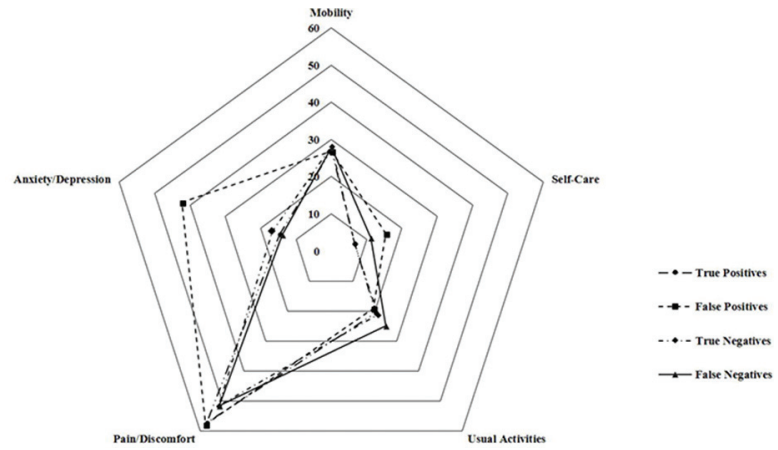

Figure 1. EuroQol-5D in patients with CHIKV infection from the Colombian COPCORD cohort.

Disclosure of Interests: None declared

DOI: 10.1136/annrheumdis-2019-eular.1603

\section{SAT0459 DIAGNOSIS AND MANAGEMENT OF ACUTE HOT JOINTS AT A TERTIARY RHEUMATOLOGY CENTRE OVER A 7-MONTH PERIOD}

Mrinalini Dey ${ }^{1}$, Pankaj Lal ${ }^{2}$, Robert N. Thompson ${ }^{1}$, Nicola Goodson ${ }^{1} .{ }^{1}$ Aintree University Hospital, Rheumatology, Liverpool, United Kingdom; ${ }^{2}$ Aintree University Hospital, Microbiology, Liverpool, United Kingdom

Background: The acute hot joint presentation is common in clinical practice, often due to crystal arthritis or non-inflammatory conditions. The most serious diagnosis is septic arthritis (SA), which can destroy cartilage within days, and therefore must be promptly diagnosed or excluded. The mortality rate for $S A$ is $7-15 \%$, despite antibiotic use [1]. Crystal arthropathies and $S A$ are difficult to distinguish clinically, requiring arthrocentesis and synovial fluid (SF) analysis, in keeping with national guidelines.

Objectives: 1. Quantify number of acute hot joint presentations to our hospital over 7 months, including incidence of SA and crystal arthropathy. 2. Determine clinical features and outcomes (including morbidity/mortality) in SA.

3. Compare clinician diagnosis with laboratory diagnosis (gold-standard diagnostic test; crystal microscopy and culture).

4. Determine the correlation between WCC and crystal arthropathy or SA Methods: We retrospectively analysed laboratory diagnostic data and elec tronic clinical records, for patients presenting to a tertiary rheumatology centre in Northwest England with an acute hot joint, February-August 2018. For crystal arthropathy and SA, sensitivity, specificity, likelihood ratios, and accuracy of diagnosis were calculated for clinician diagnosis when compared to laboratory diagnosis, using the statistical software 'R'. WCCs were used to create receiver operating curves (ROCs), with area under the curve and predicted diagnostic threshold for SA and crystal arthropathy. In cases of SA, organism, serum inflammatory markers, and outcomes were recorded.

Results: 137 patients were included. The most common site of arthrocentesis was the knee $(n=108)$. There were 53 clinician-confirmed cases of crystal arthropathy. Compared to laboratory crystal microscopy, this yielded a sensitivity of $91.7 \%$, specificity $80.2 \%$, diagnostic accuracy $83.2 \%$. There were 10 cases of native joint SA (plus 2 prosthetic and 1 case of osteomyelitis). SF cultures were positive in $80 \%$ of cases, all of which grew Staphylococcus aureus. $50 \%$ had commenced antimicrobials prior to arthrocentesis. 8 cases were treated successfully (5 prolonged admission due to underlying rheumatological disease); the 2 culture-negative cases had significant morbidity, with persistent symptoms. For SA mean serum C-reactive protein at presentation was 152; serum WCC was 12. Clinician diagnosis, compared to culture results, yielded a sensitivity of $100 \%$, specificity $98.4 \%$, diagnostic accuracy $98.5 \%$. Figure 1 shows mean SF WCC by diagnosis; highest value was SA (WCC=136293). ROC analysis of WCC yielded potential diagnostic threshold of 36450 for $S A \quad(A \cup C=0.7)$ and 10000 for crystal arthropathy $(\mathrm{AUC}=0.7)$ in our cohort.

Conclusion: The acute hot joint continues to be a common presentation in the acute medical setting. SA has considerable morbidity associated with it, especially in the presence of co-existing rheumatological disease. Our results confirm the importance of prompt arthrocentesis, prior to commencement of antimicrobials. SF WCC may also be utilised as a rapid diagnostic marker, especially differentiating between inflammatory and noninflammatory causes of a hot joint, when taken with clinical assessment.

\section{REFERENCES}

[1] Margaretten ME, et al. Does this adult patient have septic arthritis? JAMA 2007 Apr;297(13):1478-88.

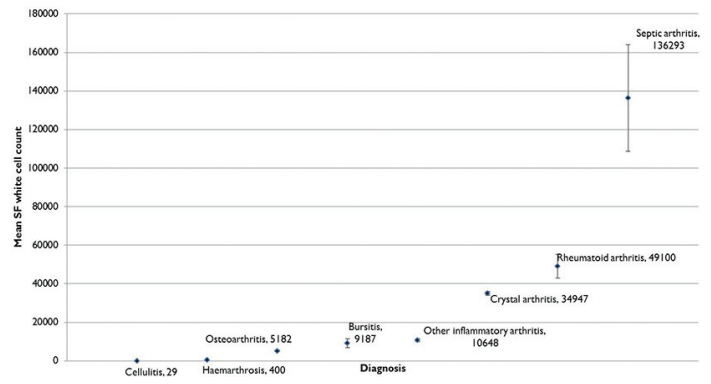

Figure 1

Disclosure of Interests: Mrinalini Dey: None declared, Pankaj Lal: None declared, Robert N. Thompson : None declared, Nicola Goodson Grant/ research support from: Research support grant from Novartis, Speakers bureau: Paid speaker UCB

DOI: 10.1136/annrheumdis-2019-eular.3484

\section{SAT0460 HCV-RELATED MIXED CRYOGLOBULINAEMIA IN THE DIRECT-ANTIVIRAL AGENTS ERA: IS THERE ADVANTAGE IN SEQUENTIAL THERAPY WITH RITUXIMAB?}

Maria Di Cicco ${ }^{1}$, Davide Antonio Filippini ${ }^{1}$, Federica Filippini ${ }^{2}$, Laura Belloli ${ }^{1}$, Valeria Campanella ${ }^{1}$, Cinzia Casu ${ }^{1}$, Michel Chevallard ${ }^{1}$, Marina Muscarà ${ }^{1}$, Mariaeva Romano ${ }^{1}$, Emanuela Schito ${ }^{1}$, Elisa Verduci ${ }^{1}$, Massimo Puoti ${ }^{3}$, Maria Vinci ${ }^{4}$, Oscar Massimiliano Epis ${ }^{1} .{ }^{1}$ Grande Ospedale Metropolitano Niguarda, Rheumatology, Milano, Italy; ${ }^{2}$ Università Politecnico, Mathematics, Milano, Italy; ${ }^{3}$ Grande Ospedale Metropolitano Niguarda, Infectious Diseases, Milano, Italy, ${ }^{4}$ Grande Ospedale Metropolitano Niguarda, Hepatology, Milano, Italy

Background: Mixed Cryoglobuminaemia(MC) is a small vessel vasculitis characterized by the presence of a monoclonal rheumatoid factor(RF) and polyclonal immunoglobulins that precipitate at cold temperature, with a large spectrum of clinical manifestations. $M C$ is strongly associated with hepatitis $\mathrm{C}(\mathrm{HCV})$ infection $>90 \%$. The new direct-acting antivirals(DAA) against $\mathrm{HCV}$, which can eradicate the virus in $>95 \%$ of patients, are supposed to have benefits on clinical manifestations of $M C$, nonetheless the amount of data is still limited. Patients with severe vasculitis may require anti-CD20 agent Rituximab(RTX) over DAA. However, despite the strong rationale, the advantage of dual therapy is still debated and the optimal therapeutic schedule unknown.

Objectives: To investigate the impact of DAA treatment on clinical manifestations and biochemical parameters of disease activity in HCV-related MC patients who reached complete viral eradication, and if pre-treatment with RTX was able to define a subset of patients with a different outcome.

Methods: Data was collected from 31 patients with MC, mean age 68 yrs, female $80 \%$, mean disease duration 10 yrs who were treated with 
DAA and reached complete viral eradication, at 3 time points: before starting DAA (pre-DDA), after completing DAA (post-DAA), 52 weeks after completing DAA $(52 w)$. Additionally, a population of 9 patients with the same disease (mean age 65 yrs, female 77\%, mean disease duration 9 yrs) but treated with one or more cycles of RTX prior receiving DAA, was analysed at the same timepoints and additionally before starting RTX(pre-RTX). At each time point clinical data (presence/absence of purpura, neuropathic manifestations, arthralgia, fatigue, nephritis) and biochemical parameters (\% cryoglobulins, C3, C4 and RF) were recorded. The change in clinical and biochemical parameters at different timepoints were evaluated.

Results: Among patients treated with DAA only, the prevalence of clinical manifestations before starting treatment was the following: purpura $31 \%$, neuropathic manifestations 52\%,arthralgia $28 \%$,fatigue $14 \%$, nephritis $3 \%$. We observed a significant reduction in purpura after treatment (pre-DDA vs post-DAA $=31 \%$ vs $13 \%, p=0.044$ ) that persisted at $52 \mathrm{w}$ (pre-DAA vs $52 \mathrm{w}=31 \%$ vs $0 \%, \mathrm{p}=0.004 \%$ ). This in parallel with a significant reduction in the level of cryoglobulins (pre-DAA vs $52 \mathrm{w}=3.99 \pm 5.28$ vs $1.6 \pm 1.7$, $\mathrm{p}=0.029$ and pre-DAA vs $52 \mathrm{w}=3.99 \pm 5.28$ vs $0.92 \pm 1.75, \mathrm{p}=0.008)$. Among patients pre-treated with RTX, the prevalence of clinical manifestations before starting RTX was: purpura $78 \%$, neuropathic manifestations $100 \%$,arthralgia $44 \%$,fatigue $50 \%$,nephritis $22 \%$; and before starting DAA was: purpura $22 \%$, neuropathic manifestations $100 \%$,arthralgia $0 \%$,fatigue $0 \%$,nephritis $0 \%$. A significant reduction in the rate of purpura (pre-RTX vs pre-DAA $=78 \%$ vs $22 \%, p=0.009 \%)$ and arthralgia (44\% vs $0 \%$, $\mathrm{p}=0.012 \%$ ) after RTX treatment was observed, but no difference in any clinical parameter when comparing pre-RTX with post-DAA and 52w. No significant change in any of the serological parameter was observed.

Conclusion: DAA treatment is associated with rapid and sustained decrease in the level of cryoglobulins and related clinical improvement, specifically purpura. Patients who had been pre-treated with RTX showed clinical improvement in terms of purpura and arthralgia, albeit transient; subsequent treatment with DAA did not result in any immediate or long term additional clinical benefit.

\section{REFERENCES}

[1] Mazzaro C. Clin Exp Rheum 2017

Disclosure of Interests: Maria Di Cicco: None declared, Davide Antonio Filippini: None declared, Federica Filippini: None declared, Laura Belloli: None declared, Valeria Campanella: None declared, Cinzia Casu: None declared, Michel Chevallard: None declared, Marina Muscarà: None declared, Mariaeva Romano Speakers bureau: janssen, Emanuela Schito: None declared, Elisa Verduci: None declared, Massimo Puoti: None declared, Maria Vinci: None declared, Oscar Massimiliano Epis Speakers bureau: BMS

DOI: 10.1136/annrheumdis-2019-eular.7781

\section{SAT0461 VERTEBRAL OSTEOMYELITIS IN THE IMMUNOSUPPRESSED PATIENTS}

Jorge Juan Fragio-Gil ${ }^{1}$, Roxana Gonzalez Mazario ${ }^{1}$, Jose Ivorra Cortés ${ }^{1}$, Francisco Miguel Ortiz Sanjuan ${ }^{1}$, Elena Grau García ${ }^{1}$, Cristobal Pávez Perales ${ }^{1}$, Marta De la Rubia Navarro ${ }^{1}$, Inmaculada Chalmeta Verdejo ${ }^{1}$, Luis Gonzalez Puig ${ }^{1}$, Isabel Martínez Cordellat ${ }^{1}$, Rosa Negueroles Albuixech ${ }^{1}$, Cristina Alcañiz Escandell ${ }^{1}$, Jose Eloy Oller Rodríguez ${ }^{1}$, Elvira Vicens Bernabeu ${ }^{1}$, Carmen Nájera Herranz ${ }^{1}$, Inés Cánovas Olmos ${ }^{1}$, María Tasias Pitarch ${ }^{2}$, Eva Calabuig Muñoz ${ }^{2}$, Miguel Salavert Lleti ${ }^{2}$, Jose Andrés Román Ivorra ${ }^{1} .{ }^{1}$ Rheumatology Department. HUP La Fe, Valencia, Spain; ${ }^{2}$ Infectious Diseases Unit. HUP La Fe, Valencia, Spain

Background: Vertebral Osteomyelitis (VO) is an infectious disease that could involve intervertebral space (discitis). Its incidence is raising for several reasons, such as the increasing number of patients on immunosuppressive therapy. The treatment includes long term antibiotic, that should be initiated after biopsy (if possible) and sometimes, further surgery is needed ${ }^{1}$.

Objectives: To analyze the influence on prognosis of detection of $\mathrm{OV}$ in immunosuppressed (IS) patients.

Methods: Single center retrospective observational study including IS adult patients diagnosed of $\mathrm{VO}$ based on the combination of clinical presentation with either a definitive bacteriologic diagnosis or pathological and/or imaging studies from January 2010 to January 2019. Demographic features, concurrent diseases, clinical history (length of pain and fever prior to admission), laboratory findings, microbiological diagnosis and radiological data were compiled. We considered as IS patients those who had rheumatic or inflammatory bowel disease undertaking immunomodulatory drugs, solid organ transplantation receptors, patients with an active malignancy or Human Immunodeficiency Virus (HIV) infected. Clinical and radiological history of lumbar stenosis or disc herniation was considered as prior spine pathology. Surgical infections were excluded. We considered deaths attributive to PVO those which were directly caused by the infectious picture and/or its complications during the next year after diagnosis.

Results: Eighteen of 122 patients with VO (21.96\%) were IS. Basal demographic and clinical features are exposed in table 1. Detailed data about the cause of the IS is provided separately in table 2. Duration of pain prior to diagnosis had a median of 30 days $(15.5,55)$. C reactive protein showed a median value of $65.2 \mathrm{mg} / \mathrm{L}(19.32,153.9)$ and Erythrocyte Sedimentation Rate mean value was $80.57 \mathrm{~mm} / \mathrm{H}$ (31.75). MRI was the imag ing technique most often used for diagnosis (83.33\%), followed by CT scan $(16.67 \%)$. The region most frequently affected was lumbosacral spine (44.44), then dorsal (33.33\%) and finally cervical $(22.22 \%)$. Some abscess (epidural or paravertebral) was detected in 16 patients $(88.89 \%)$ close to non-IS patients $(90.38 \%)$. Worst data concerning vertebral destruction was observed $(66.67 \%$ in IS group against 49.04 in non-IS, $p=0.3$ ) and cord compression $(61.11 \%$ versus $26.92 \%$ respectively, $\mathrm{p}=0.05)$. Fifteen patients underwent CT guided biopsy (83.33\%) with prior antibiotic exposure in 11 of them. Median exposure was 4.5 days $(3,8.75)$. Delay from admission to procedure had a median value of 6 days $(3.5,9)$. Culture was positive in $53.33 \%$ of cases. In 10 patients, the picture was attributed to Gram+ $(55.56 \%)$, in other 2 cases Gram- (11.11) and 1 case of tuberculosis (5.56). In 5 cases (27.78\%) final pathogen was unknown. Four patients (22.22\%) required further surgery and 2 patients (11.11) died, similar than non-IS group, $11.54 \%$.

Conclusion: In general terms, data about imaging is worse in IS patients and higher proportion of cervical spine involvement was also noted. Although, early intervention (diagnosis, punction guided biopsy and treat ment) seems to be protective against a bad outcome, since IS patients showed similar prognosis (further surgical procedures and death) than non IS patients. To sum up, new onset back pain in a IS patient, should be thoroughly studied so as to consider a VO as soon as possible.

Disclosure of Interests: None declared

DOI: 10.1136/annrheumdis-2019-eular.6621

\section{SAT0462 INTEREST OF FOLLOW-UP IMAGING EXAMINATIONS IN PATIENTS WITH PYOGENIC VERTEBRAL OSTEOMYELITIS: A RETROSPECTIVE STUDY}

Sophie Hecquet, Kevin Bouiller, Frank Verhoeven, Clément Prati, Daniel Wendling, Catherine Chirouze. Centre hospitalier régional universitaire de Besançon, Besançon, France

Background: Systematic follow-up imaging in patients with pyogenic vertebral osteomyelitis (PVO) is widespread. However, it is discussed, and there is no recommendation.

Objectives: Evaluate the interest of follow-up imaging examinations in patients with pyogenic vertebral osteomyelitis

Methods: We conducted a retrospective cohort analysis of patients with PVO who had both baseline and follow-up imaging results available in a French university hospital during the period of 2010-2018. We have classified the follow-up images into two groups, improvement/stability and deterioration, compared with the baseline findings. For each patient, we compared their imaging follow-up to their clinical-biological condition assessed at the same time.

Results: We have collected 80 patients. The median age was 71 years [32-89], 46 men, 13 patients had a history of spinal surgery. The mos frequently reported germ was methicillin-sensitive staphylococci and the level of spinal involvement was predominantly lumbar. A Computerized Tomography (CT) was performed in $64 \%$ and a Magnetic Resonance Imaging (MRI) in $85 \%$ at the time of diagnosis. We identified 89 followup images, 58 MRIs, 31 CTs. The median delay of realisation was 85 days [11-364]. Soft tissue infiltration was observed in 50 patients compared to 26 on follow-up images but 3 were new. Similarly, $24 \%$ of the initial images had epiduritis compared to $16 \%$ during follow-up, $3 \%$ had appeared secondarily. There were $12 \%$ initial erosions described com pared to $25 \%$ at follow-up. Of the 33 patients with clinical and biological recovery, $67 \%$ of follow-up images were classified as improving/stable and $33 \%$ as worsening (new abscesses $(n=3)$, extension of soft tissue infiltration $(n=2)$ and/or epiduritis $(n=2)$ or appearance of new locations $(n=2))$. Among the 37 patients considered as unhealed, $87 \%$ of follow-up images were classified as improving/stable and $13 \%$ as worsening (new abscesses $(n=1)$, extension of soft tissue infiltration $(n=1)$ and/or epiduritis $(n=1)$ or appearance of new locations $(n=1))$. 